\title{
Modelado y Control de un Sistema Híbrido de Generación de Energía Eléctrica
}

Omar Aguilar ${ }^{1}$

\section{Resumen}

Con la creciente preocupación en materia de energía, el desarrollo de fuentes de energía renovables es cada vez más atractivo. Este trabajo presenta la regulación de la potencia de salida de un sistema de generación eólica basado en un generador síncrono de imanes permanentes. La sincronización a la red eléctrica es un punto fundamental para lograr la interconexión de sistemas híbridos utilizando dispositivos basados en electrónica de potencia. En este trabajo se propone un esquema de control PID para el seguidor de fase, con el objetivo de mantener la frecuencia de la corriente generada igual a la del sistema eléctrico convencional. Las corrientes de la fuente inversora de voltaje son controladas en un marco de referencia ortogonal dqo usando un compensador por retroalimentación en adelanto. Las simulaciones realizadas en MATLAB / Simulink, demuestran que los controladores presentan un excelente desempeño en estado transitorio, así como en estado estacionario.

Palabras Clave: Control de corriente, generación distribuida, fuente inversora de voltaje. 


\section{Abstract}

Due to energy growing concerns, the development of renewable energy sources is becoming more appealing. This paper presents the power output regulation of a wind power generation system based on a permanent magnet synchronous generator. Synchronization to the mains supply is fundamental to achieve interconnection of hybrid systems using devices based on power electronics. This paper proposes a PID control scheme for the phase tracker in order to maintain the generated current frequency equal to the conventional power system. Currents of voltage source inverter are controlled in a COD orthogonal reference frame, using a feedback compensator in advance. Simulations performed in MATLAB / Simulink show that the controllers have an excellent performance in the transient state and steady state.

Keywords: Current control, distributed generation, voltage source inverter. 


\section{Introducción}

En los últimos años, la energía eólica se considera como la fuente de energía renovable más importante para la generación de energía eléctrica (Master, 2004). La cantidad de energía eólica capturada depende principalmente de las condiciones meteorológicas de cada lugar en específico. El costo de producción de la energía eléctrica es fijo una vez que la planta de generación es construida (Munteanu, Bratcu, Cutululis and Ceanga, 2008). En las últimas décadas, el desarrollo de esta tecnología ha tenido un gran avance, debido principalmente al incremento en el precio de los combustibles fósiles, las bajas reservas existentes, y al impacto adverso sobre el medio ambiente (Master, Munteanu et al. y Wu, Lang, Zargari y Kouro, 2011).

En la actualidad existen diversas configuraciones de turbinas eólicas. Los generadores eólicos se diferencian por el tipo de generador eléctrico, el esquema para obtener la máxima potencia del viento y la técnica de disparo de los dispositivos basados en electrónica de potencia (Munteanu et al.).

La turbina eólica es el dispositivo encargado de extraer la energía disponible en el viento (Hau, 2006). Existen diversos tipos de generadores eléctricos que pueden ser acoplados a turbinas eólicas. En este trabajo se elige un Generador Síncrono de Imanes Permanentes (GSIP) porque es una excelente opción para los sistemas de generación de baja potencia. La máquina síncrona posee alta eficiencia, es libre de mantenimiento y el acoplamiento a la red eléctrica es fácil y económico (Ion Boldea, 2006).

Recientemente se ha incrementado el interés en la teoría de control no lineal en los sistemas eléctricos y electrónicos, particularmente para interconectar sistemas de generación distribuidos a la red eléctrica (Yazdani e Iravani, 2010). Las técnicas no lineales aumentan la robustez de los controladores, mejorando el comportamiento dinámico del sistema. Sin embargo, poseen una estructura compleja que dificultan su implementación, comparada con los controladores basados en modelos linealizados. Además, se requiere la estimación de parámetros del sistema para cancelar las no linealidades inherentes, incrementando la complejidad en el análisis de estabilidad. Con el avance en tecnología de estado sólido y dispositivos inteligentes es posible superar parte de las desventajas mencionadas anteriormente (Esram, 2007 y Bialasiewicz, 2008).

En este trabajo se emplean técnicas de control lineal y no lineal (Utkin, Guldner y Shi, 2009), para regular las diferentes etapas del sistema, y satisfacer los requerimientos de potencia activa y reactiva de la red eléctrica. El sistema de generación distribuido está compuesto por una turbina eólica, un GSIP, un rectificador $\mathrm{CA}-\mathrm{CD}$, un convertidor de $\mathrm{CD}-\mathrm{CD}$ elevador, un seguidor de fase y una fuente inversora de voltaje, como se muestra en la Figura 1. 


\section{Energía Eólica}

La estimación de la velocidad del viento por medio un modelo matemático, permite obtener diferentes secuencias de velocidades, ya sea con características deseadas o aproximadas a las mediciones en la región de estudio. El modelo empleado en este trabajo, considera la suma de cuatro componentes (Ackerman, 2005): a) valor promedio, vwa, b) componente en rampa, representando un incremento estable en la velocidad, $\operatorname{vwr}(t), c)$ elemento ráfaga, $\operatorname{vwg}(t), y d)$ componente de turbulencia, $\operatorname{vwt}(\mathrm{t})$. La representación general de la velocidad del viento y sus componentes, se muestra en (1):

$$
v_{w}(t)=v_{w a}+v_{w r}(t)+v_{w g}(t)+v_{w t}(t)
$$

\section{Velocidad promedio del viento}

El valor promedio de la velocidad del viento (2), se obtiene a partir de la sumatoria de los valores de velocidad del viento, multiplicados por la probabilidad de presentarse, como sigue:

$$
v_{w a}=\int_{0}^{\infty} v f(v) d v
$$

donde $\mathrm{f}$ (v) es la función de densidad de probabilidad de Weibull (3) y v es la velocidad del viento en $\mathrm{m} / \mathrm{s}$. La función de densidad de probabilidad, proporciona la posibilidad de que cierta velocidad del viento se presente en la región ( $\mathrm{Wu}$, et al.).

$$
f(v)= \begin{cases}\frac{k}{c^{k}}\left(v^{k-1}\right) e^{\left[-y^{k}\right]} & v \geq 0 \\ 0 & v<0\end{cases}
$$

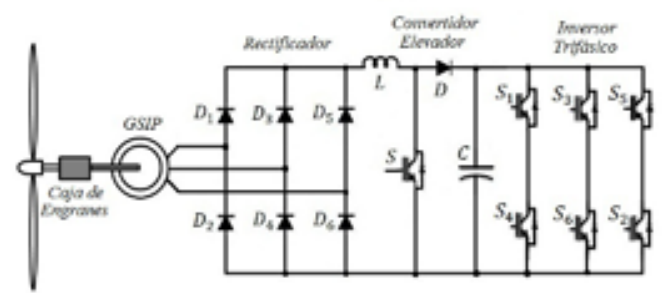

Fig. 1 Sistema de generación distribuido

\section{Componente Rampa del Viento}

La componente rampa del viento (4), representa un incremento o decremento estable de la velocidad. Está definida por tres parámetros, la amplitud de la rampa en $\mathrm{m} / \mathrm{s}$; el tiempo de inicio de la rampa Tsr y el tiempo de término de la rampa Ter, ambos expresados en segundos. 


\section{Componente Rampa del Viento}

La componente rampa del viento (4), representa un incremento o decremento estable de la velocidad. Está definida por tres parámetros, la amplitud de la rampa $\hat{\mathrm{A}}_{\mathrm{r}}$ en $\mathrm{m} / \mathrm{s}$; el tiempo de inicio de la rampa $\mathrm{T}_{\mathrm{sr}}$ y el tiempo de término de la rampa $\mathrm{T}_{\mathrm{er}}$, ambos expresados en segundos.

$$
v_{W r}(t)=\left\{\begin{array}{rc}
t<T_{S} & v_{W r}=0 \\
T_{S S} \leq t \leq T_{e r} & v_{W r}=\hat{A}_{r} \frac{\left(t-T_{S r}\right)}{\left(T_{e r}-T_{S}\right)} \\
T_{e r}<t & v_{W r}=\hat{A}_{r}
\end{array}\right.
$$

\section{Componente Ráfaga del Viento}

La componente ráfaga del viento, proporciona un incremento rápido con variación significativa entre el pico y la parte estable de la curva. Se caracteriza por tres parámetros, la amplitud de la ráfaga $\hat{\mathrm{A}}_{\mathrm{g}}$ en $\mathrm{m} / \mathrm{s}$, el tiempo de inicio de la ráfaga $\mathrm{T}_{\mathrm{sg}} \mathrm{y}$ el tiempo de término $\mathrm{T}_{\mathrm{eg}}$ en segundos.

$$
v_{w g}(t)=\left\{\begin{array}{c}
t<T_{s g} \quad v_{w r}=0 \\
T_{s g} \leq t \leq T_{e g} \quad v_{w r}=\hat{A}_{g}\left\{1-\cos \left[2 \pi\left(\frac{t-T_{s g}}{T_{e g}-T_{s g}}\right)\right]\right\} \\
T_{e g}<t \quad c=0 \\
v_{w r}=0
\end{array}\right.
$$

\section{Turbulencia del Viento}

En este trabajo se utiliza el modelo espectral de Kaimal (Ackermann, 2005) por su mejor comportamiento en para estimar el efecto de turbulencia atmosférica. La turbulencia del viento, presenta un comportamiento aleatorio, para obtenerla se utiliza la densidad espectral de potencia $\mathrm{S}_{\mathrm{wt}}(\mathrm{f})(7)$.

$$
\begin{aligned}
& v_{w t}(t)=\sum_{i=1}^{n} \sqrt{\mathcal{S}_{w t}(f) \Delta f} \cos \left(2 \pi f t+\varphi_{i}\right) \\
& \frac{f S_{w t}(f)}{\sigma^{2}}=\frac{4 f L_{t} / v_{w a}}{\left(1+6 f L_{t} / v_{w a}\right)^{5 / 3}}
\end{aligned}
$$

\section{Sistema de Generación Distribuido}

A continuación se describen las ecuaciones dinámicas, de cada uno de los elementos que componen al sistema de generación distribuida 


\section{Aerodinámica de la Turbina Eólica}

La potencia mecánica captada por la turbina eólica se puede calcular como sigue

$$
P_{w t}=0.5 \rho A v_{w}^{3} C_{P}(\lambda)
$$

el par de la turbina eólica (9), se obtiene entre la relación de potencia extraída y la velocidad angular del rotor $\left(\omega_{1}\right)$.

$$
\Gamma_{w t}=\frac{P_{w t}}{\omega_{I}}=\frac{1}{2} \pi \rho v^{2} R^{3} C_{\Gamma}(\lambda)
$$

el coeficiente $\mathrm{C}_{\Gamma}(\lambda)(10)$, representa el par de salida de la turbina eólica, expresado por

$$
c_{\Gamma}(\lambda)=a_{0}+a_{1} \lambda+a_{2} \lambda^{2}
$$

donde $\mathrm{a}_{\mathrm{i}}=0,1,2$, son determinados por tablas y $\lambda$ es la velocidad punta de la turbina eólica.

\section{Modelo Dinámico del GSIP}

Se emplea el modelo dinámico del GSIP presentado en (Boldea, 2006)

$$
\begin{aligned}
& v_{d}=-R_{S} j_{d}-L_{d} \frac{d i_{d}}{d t}+L_{q} j_{q} \omega_{s} \\
& v_{q}=-R_{S} j_{q}-L_{q} \frac{d i_{q}}{d t}+\left(L_{d} d_{d}-\psi_{m}\right) \omega_{s}
\end{aligned}
$$

donde $R_{s}$ es la resistencia del estator en ohms; $L_{d}$ y $L_{q}$ son las inductancias del eje $d$ y el eje $q$ respectivamente en henrios; $\Psi_{\mathrm{m}}$ enlaces de flujo del imán permanente en webers y $\omega_{\mathrm{s}}$ es la velocidad angular del estator en $\mathrm{rad} / \mathrm{s}$. La dinámica del sistema mecánico del GSIP (13), se puede expresar como sigue

$$
\begin{aligned}
& v_{d}=-R_{s} j_{d}-L_{d} \frac{d i_{d}}{d t}+L_{q} i_{q} \omega_{s} \\
& v_{q}=-R_{s} j_{q}-L_{q} \frac{d i_{q}}{d t}+\left(L_{d} j_{d}-\psi_{m}\right) \omega_{s}
\end{aligned}
$$

donde $\Gamma_{\mathrm{wt}}$ es el par del viento en $\mathrm{Nm}(10) ; \Gamma_{\mathrm{g}}$ es el par electromagnético en $\mathrm{Nm}$ (14) y $J_{\text {eq }}$ es la inercia rotacional del generador en $\mathrm{kg}^{*} \mathrm{~m} 2$. 


\section{Rectificador Trifásico no Controlado}

El rectificador trifásico no controlado se muestra en la Fig. 2. El arreglo de diodos convierte una señal de corriente alterna, que proviene del GSIP en una señal de corriente continua. El voltaje rectificado es igual a la diferencia entre el máximo de la fase A y el mínimo de la fase B (Nehrir y Wang, 2009).

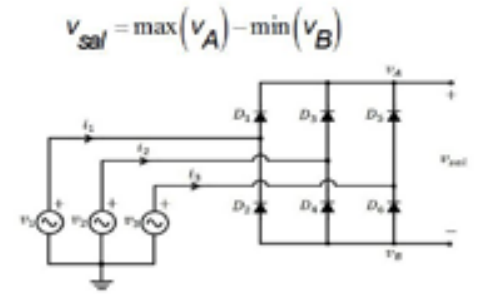

Fig. 2 Diagrama esquemático del rectificador trifásico

\section{Convertidor de CD-CD Elevador}

El convertidor CD-CD elevador, tiene la función de entregar un voltaje mayor al voltaje de entrada. En la Fig. 3 se muestra el diagrama esquemático del convertidor elevador. Su modelo dinámico (Pejovic, 2007) se obtiene realizando un análisis con interruptor abierto y cerrado, obteniendo (16) y (17)
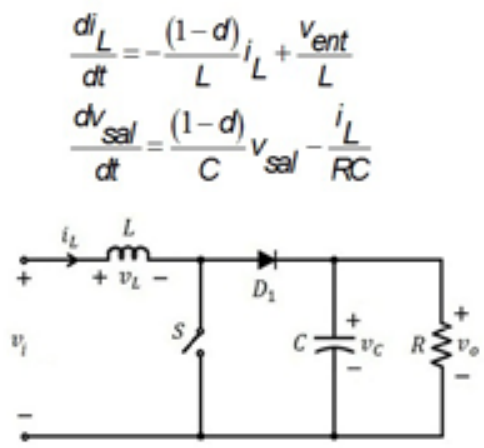

Fig. 3 Diagrama esquemático del convertidor de CD-CD elevador

Fuente inversora de voltaje

El modelo matemático derivado del análisis de cada una de las mallas del circuito mostrado en la Fig. 4, se obtiene (18-20) (Utkin et al., 2009).

$$
\begin{aligned}
& L \frac{d i_{a}}{d t}+R i_{a}+v_{a s}=H_{1} v_{d c}-v_{n} \\
& L \frac{d i_{b}}{d t}+R j_{b}+v_{b s}=H_{3} v_{d c}-v_{n} \\
& L \frac{d i_{c}}{d t}+R i_{c}+v_{c s}=H_{5} v_{d c}-v_{n}
\end{aligned}
$$


donde $R$ es la resistencia de carga en ohms; $L$ es la inductancia del filtro en henrios; $\mathrm{v}_{\mathrm{dc}}$ es el voltaje de entrada en volts; $\mathrm{v}_{\mathrm{is}}$ es el voltaje de la red eléctrica; $\mathrm{H}_{1}, \mathrm{H}_{3}$ y $\mathrm{H}_{5}$ son las señales de control de los dispositivos de conmutación y $v_{n}$ es el voltaje en el neutro. El voltaje $\mathrm{v}_{\mathrm{n}}$ se obtiene por (21).

$$
v_{n}=\frac{v_{d c}}{3}\left(H_{1}+H_{3}+H_{5}\right)
$$

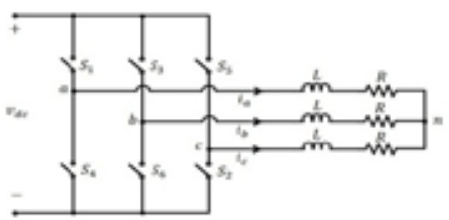

Fig. 4 Esquema de la fuente inversora de voltaje

Se pude observar que el modelo (18-21) es no lineal. Para facilitar el diseño del esquema de control, se transforma a un sistema rotatorio ortogonal síncrono empleando $w$ y la transformada de Park (Yazdani, 2010). El modelo dinámico (2223) en marco de referencia dqo a partir de (18-21) es

$$
\begin{aligned}
& L \frac{d_{i d}}{d t}=-R_{d}+L w_{q}+d_{d} v_{d c}-v_{d} \\
& L \frac{d_{i q}}{d t}=-R d_{q}+L w_{d}+d_{q} v_{d c}-v_{q} \\
& \frac{d v_{d c}}{d t}=-\frac{d_{d}}{C} i_{d}-\frac{d_{q}}{C} i_{q}+\frac{i}{C}
\end{aligned}
$$

\section{Diseño del Esquema de Control}

Para suministrar a la carga una señal de voltaje dentro de parámetros especificados en los códigos de red. Por lo tanto, se requieren de diferentes algoritmos de control, que obliguen a cada subsistema a entregar los valores deseados en cada etapa del sistema.

\section{Control del convertidor de CD-CD elevador}

Partiendo del modelo no lineal del convertidor elevador (16) y (17) se le induce un controlador no lineal por modos deslizantes (Yazdani, 2010). La corriente deseada $i_{L d}(25)$ se obtiene a partir de un voltaje de salida $\left(\mathrm{v}_{\text {sal }}\right)$, la función de conmutación (s) (26), es la diferencia entre la corriente de salida y la deseada. La señal de conmutación (27), permite obtener la señal de control (d), que proporciona los pulsos de disparo del IGBT del convertidor. 


$$
\begin{aligned}
& i_{L d}=\frac{v_{\text {sald }}^{2}}{R v_{\text {ent }}} \\
& s=i_{L}-i_{L d} \\
& d=\frac{1}{2}(1-\operatorname{sign}(s))
\end{aligned}
$$

\section{Control de potencia activa y reactiva}

Para suministrar la potencia activa y reactiva que requiere la carga, se emplea un esquema de control de corriente para la fuente inversora de oltaje (FIV) empleando un técnica de compensación en adelanto (Yazdani, 2010). Se miden voltajes y corrientes de la red eléctrica para calcular las potencias deseadas. De la misma forma se miden las corrientes a la salida de la FIV para generar la señal de error que entra al controlador. Todos los voltajes y corrientes se transforman al marco de $\alpha \beta$ al ángulo $\theta$ (28), que se requiere para realizar la transformación $d q o$.

$$
\theta=\angle(\alpha+j \beta)
$$

Se diseña un controlador PI para la corriente en eje $\mathrm{d}$ y otro para la corriente en eje $q$. Debido a la presencia de $L w i_{q}$ y $L w i_{d}$ en (22-23), la dinámica de $i_{d}$ e $i_{q}$ están acopladas. Para desacoplar la dinámica, se determina las señales moduladoras $m_{d} \mathrm{y} m_{q}$ como:

$$
\begin{aligned}
& m_{d}=\frac{2}{v_{d c}}\left(u_{d}-L \omega_{0} i+v_{d}\right) \\
& m_{d}=\frac{2}{v_{d c}}\left(u_{d}-L \omega_{0} i+v_{d}\right)
\end{aligned}
$$

donde $u_{d}$ y $u_{q}$ son las señales de control. Para comparar las señales moduladoras con la portadora triangular de alta frecuencia, se hace una transformación $d q 0-a b c$, para obtener $m_{a^{\prime}} m_{b}, m_{c}$. Para determinar el valor de $\omega 0$ se diseñará un seguidor de fase mediante un controlador PID.

En la Fig. 5 se observa el diagrama a bloques del esquema de control de potencia, donde se aprecia cómo se obtiene las señales moduladoras $m_{d}$ y $m_{q}$. Las señales de control son transformadas a coordenadas abc para obtener los pulsos SPWM. Las mediciones de corriente y voltaje de la red eléctrica se transforman al marco de referencia $d q 0$ mediante el ángulo $(\rho)$ que entrega el seguidor de fase. 


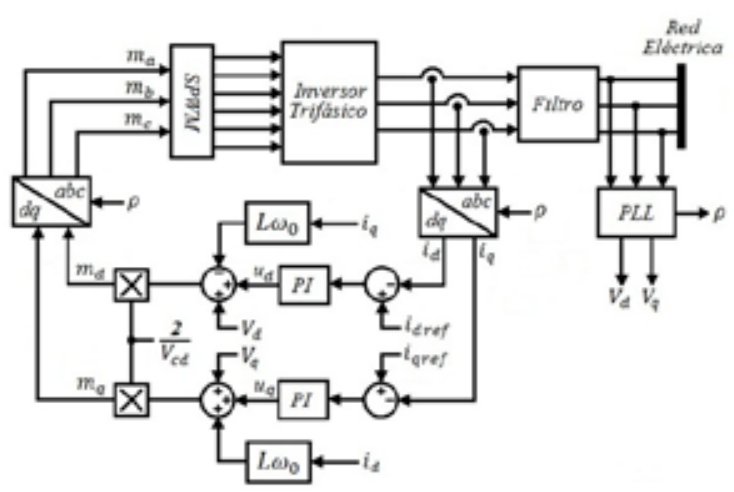

Fig. 5 Diagrama a bloques del sistema de control de potencia

\section{Seguidor de Fase (Phase-Locked Loop PLL)}

En la Fig. 6 se puede apreciar el diagrama a bloques del PLL con la estructura de control propuesta. En el diagrama se aprecia un filtro identificado por $\mathrm{H}(\mathrm{s})$ y el control PID D(s). En general, los seguidores de fase consisten de tres bloques funcionales: un oscilador controlado por voltaje, un detector de fase y un filtro. Para mantener la sincronización, el detector de fase realiza una comparación entre la señal de referencia y la salida del oscilador controlado. La señal de error generando se procesa por el filtro para el control del oscilador para obtener el mínimo error de fase. Un incremento en el error de fase produce un cambio de fase y frecuencia en la salida del oscilador controlable reduciendo el error.

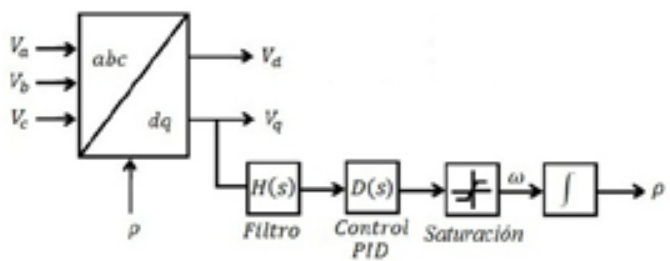

Fig. 6 Diagrama a bloques del PLL con control PID.

El control PID pretende que el sistema tenga un margen de fase de aproximadamente $\mathrm{MFd}=60^{\circ}$. Por lo tanto, el sistema tendrá una respuesta más rápida pero presentará mayores oscilaciones. Para eliminar la respuesta no deseada se implementa la acción derivativa. Para calcular los parámetros del controlador PID, se grafica el diagrama de bode de la función de transferencia del filtro H(s), Fig. 7. Con ayuda del diagrama Bode obtienen los parámetros del controlador PI.

En la gráfica se puede observar que el sistema en lazo abierto tiene una frecuencia de transición de $\omega 0 \mathrm{db}=393 \mathrm{rad} / \mathrm{s}$ y un margen de fase de $\mathrm{MF} 0=35^{\circ}$. Posteriormente se agrega la acción derivativa siguiendo el procedimiento propuesto en Golnaraghi, F. \& 
Kuo, B. C. (2010). La frecuencia de transición deseada se obtiene a partir del ángulo de la respuesta en la frecuencia de $\mathrm{H}(\mathrm{s})$ más un ángulo de remanente de fase $\theta=8^{\circ}$.

$$
\angle \mathrm{G}(\mathrm{j} \omega)=-180^{\circ}+\mathrm{MF}_{\mathrm{d}}+\theta=-180^{\circ}+60^{\circ}+8^{\circ}=-112^{\circ}
$$

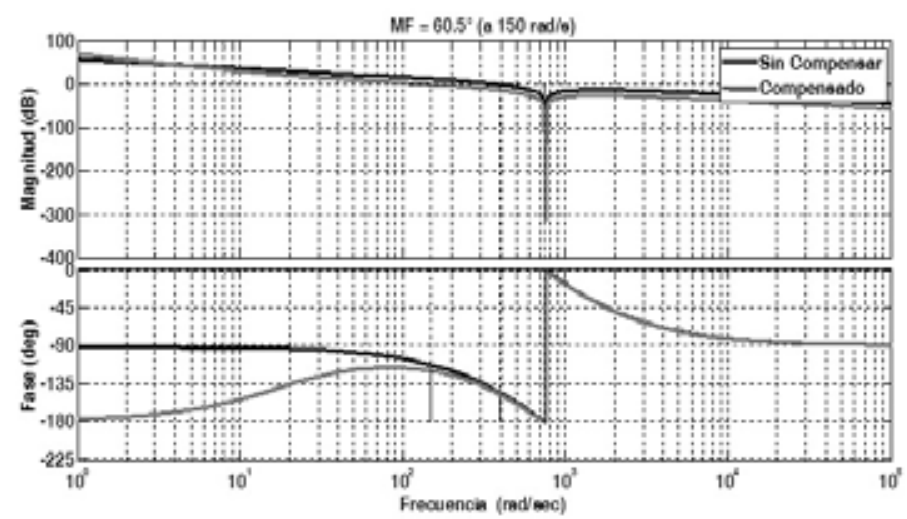

Fig. 7 Comparación entre el diagrama de bode del PLL sin compensar y compensado.

La frecuencia de transición $\omega^{\prime} 0 \mathrm{db}$, se encuentra en los $-112^{\circ}$ de la curva de fase del sistema sin compensar. La la frecuencia de transición $\omega^{\prime} 0 \mathrm{db}=149 \mathrm{rad} / \mathrm{s}$, a dicha frecuencia el sistema tiene una ganancia de $\left|\mathrm{G}\left(\mathrm{j} \omega^{\prime} 0 \mathrm{db}\right)\right|=12.6 \mathrm{~dB}$.

En la Fig. 7 se puede observar la comparación del diagrama de bode del sistema sin compensar y del sistema compensado, es posible observar que en el sistema compensado el margen de fase tiene un valor $\mathrm{MFd}=60.5^{\circ}$, muy aproximado al valor deseado.

\section{Simulaciones y Resultados}

Los modelos presentados se implementaron en Matlab/Simulink para su simulación, donde se puede observar el comportamiento del sistema ante diferentes condiciones operativas.

Tabla I Parámetros de la turbina eólica

\begin{tabular}{ll}
\hline \multicolumn{2}{c}{ Turbina eólica } \\
\hline Densidad del aire & $1.25 \mathrm{~kg} / \mathrm{m} 3$ \\
\hline Largo de las aspas & $2.5 \mathrm{~m}$ \\
\hline Velocidad del viento & $10 \mathrm{~m} / \mathrm{s}$ \\
\hline Resistencia del estator & $3.3 \Omega$ \\
\hline Inductancia del eje d y eje q & $41.56 \mathrm{mH}$ \\
\hline Flujo de los imanes permanentes & $0.4382 \mathrm{~Wb}$ \\
\hline No. de pares de polos & 3 pares \\
\hline Inercia equivalente & $0.0552 \mathrm{Kg} \mathrm{m} 2$ \\
\hline
\end{tabular}


Tabla II Parámetros del sistema de potencia

\begin{tabular}{ll}
\hline \multicolumn{2}{c}{ Convertidor CD - CD } \\
\hline Inductancia & $15.91 \mathrm{mH}$ \\
\hline Capacitor & $4.7 \mu \mathrm{F}$ \\
\hline Voltaje de salida deseado & $650 \mathrm{v}$ \\
\hline Inversor Trifásico & \\
\hline Inductancia & $25 \mathrm{mH}$ \\
\hline Resistencia & $20 \Omega$ \\
\hline Voltaje en la red & $220 \mathrm{v}$ \\
\hline
\end{tabular}

Para obtener la simulación de la turbina eólica se utilizan los datos de la Tabla I y II. En la Fig. 8 se observa una secuencia de la velocidad del viento considerando las cuatro componentes (1-7).

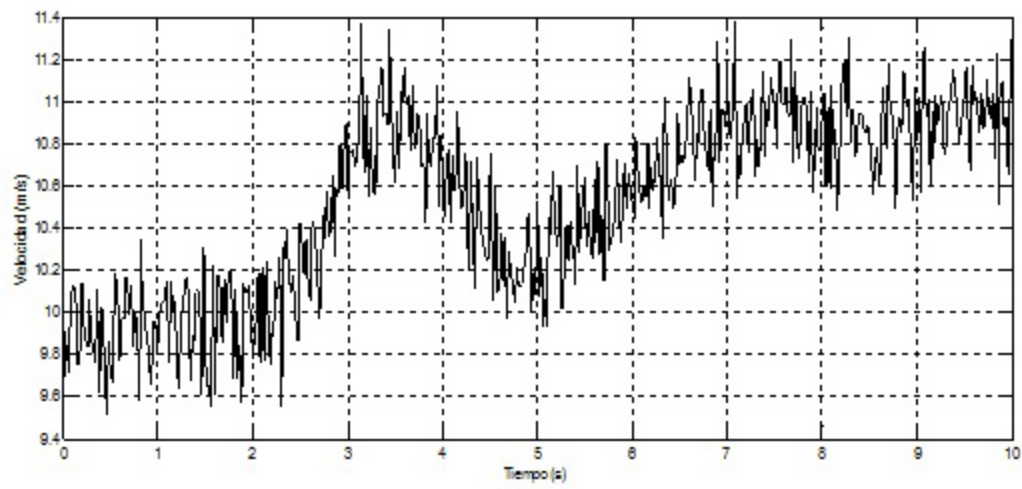

Fig. 8 Velocidad aproximada del viento

En la Fig. 9 se muestra el voltaje en las terminales del GSIP, cuando la turbina eólica transfiere un par mecánico al rotor del generador atreves de una transmisión rígida $(i=7)$.

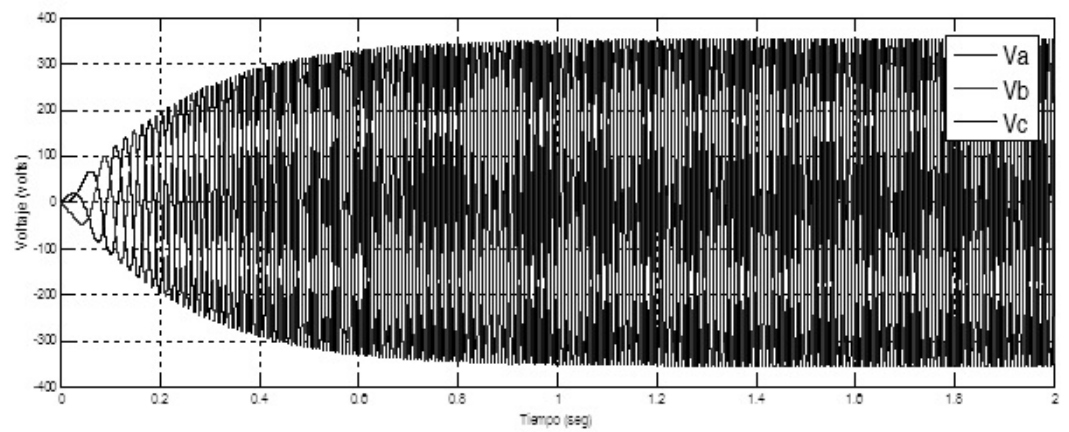

Fig. 9 Simulación de la turbina eólica con velocidad del viento constante de $10 \mathrm{~m} / \mathrm{s}$. 
Para la simulación del sistema de potencia se usan los parámetros de la Tabla II. En la Fig. 10(a) se muestra el voltaje de salida del rectificador no controlado, la Fig. 10(b) muestra el voltaje de salida del convertidor elevador. En Fig. 10(a) se puede notar que el voltaje de salida rectificado oscila entre un rango de 500 y 600 volts, este voltaje entra al convertidor CD-CD para elevarlo y mantenerlo constante en 650 volts.

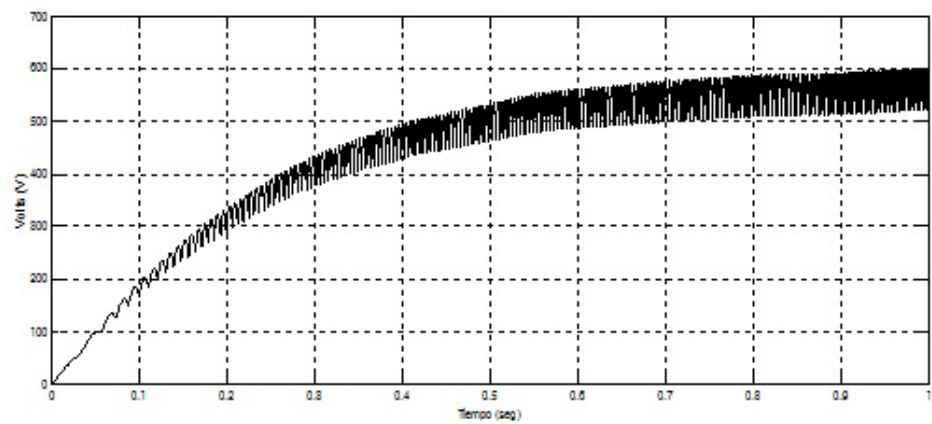

(a)

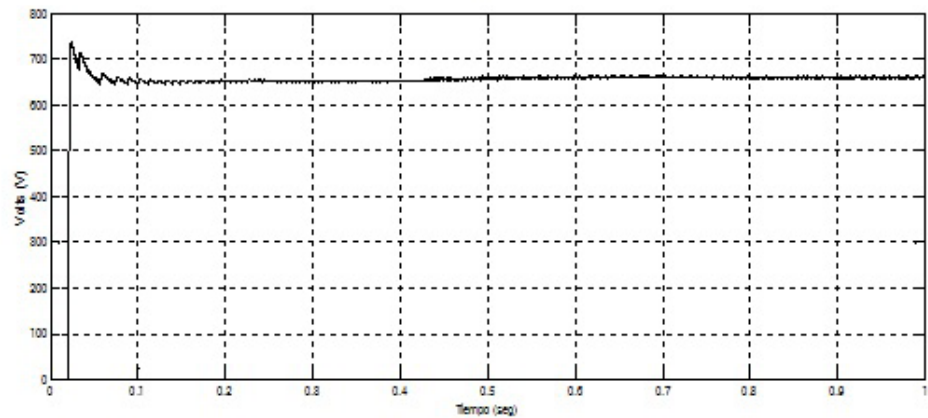

(b)

Fig. 10 Voltajes de salida de la etapa de rectificación y regulación. (a) Rectificador trifásico no controlado. (b) Voltaje de salida del convertidor de CD-CD elevador.

La Fig. 11 muestra la respuesta del esquema de control propuesto, durante el proceso de arranque y cuando existen variaciones de potencia en la red eléctrica. La principal tarea del controlador, en este trabajo, es mantener un voltaje constante.

El sistema está sujeto a la siguiente secuencia de eventos: antes de $t=0.10 \mathrm{seg}$, el controlador no funciona y se bloquean las compuertas de disparo de los IGBT. Esto permite al GSIP alcanzar su régimen en estado estable. Cuando de $t=0.10$ seg, las compuertas se desbloquean y el controlador funciona, mientras $\mathrm{P}_{\text {ref }}=1 \mathrm{Kw}$ $\mathrm{y} \mathrm{Q}_{\text {ref }}=0$. A $t=0.20 \mathrm{seg}, \mathrm{P}_{\text {ref }}$ cambia de forma abrupta de $1 \mathrm{kw}$ a $2 \mathrm{kw}$ con $\mathrm{Q}_{\text {ref }}=$ 0 . Después de $t=0.40 \mathrm{seg}, \mathrm{P}_{\text {ref }}$ cambia de forma abrupta de $2 \mathrm{kw}$ a $0.100 \mathrm{kw}$ con $\mathrm{Q}_{\mathrm{ref}}=0$, como se muestra en Fig. 11(a) y 11(b). 
La Fig. 11 y 12 muestran el desempeño y el tiempo de respuesta de la fuente inversora de voltaje durante el proceso de arranque y ante perturbaciones externas.

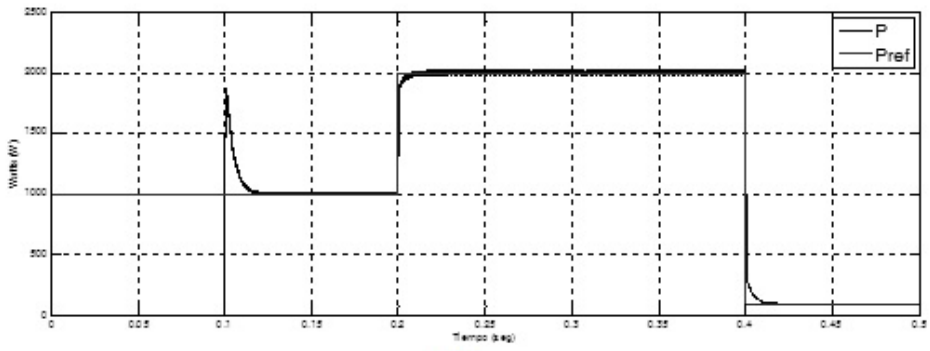

(a)

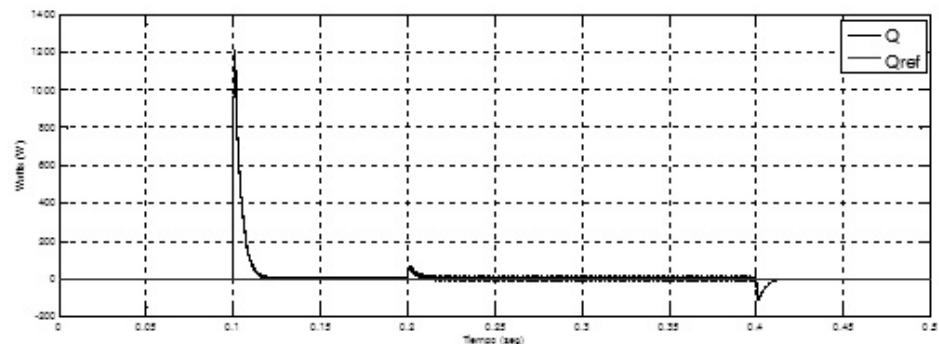

(b)

Fig. 11 Respuesta dinámica de la potencia real y reactiva, (a) Potencia real, (b) Potencia reactiva

La Fig. 11 muestra el comportamiento de las potencias activas y reactivas, se puede observar que alcanzan rápidamente el valor deseado. La Fig. 12 muestra la dinámica de la señal de corriente y voltaje durante las variaciones de potencia.

\section{Conclusiones}

El sistema de generación de energía eléctrica presentado en este trabajo, presenta un comportamiento de acuerdo a los resultados esperados, ya que entrega una señal de corriente y de voltaje que pueden ser utilizados para alimentar una carga independiente o conectarse a la red eléctrica. Es importante señalar que el algoritmo de control que se aplica al sistema, tiene un comportamiento aceptable en estado estable y en estado transitorio, porque el tiempo en alcanzar su régimen en estado estable es relativamente rápido, ante la presencia de alguna perturbación o cambio de condición de operación. 


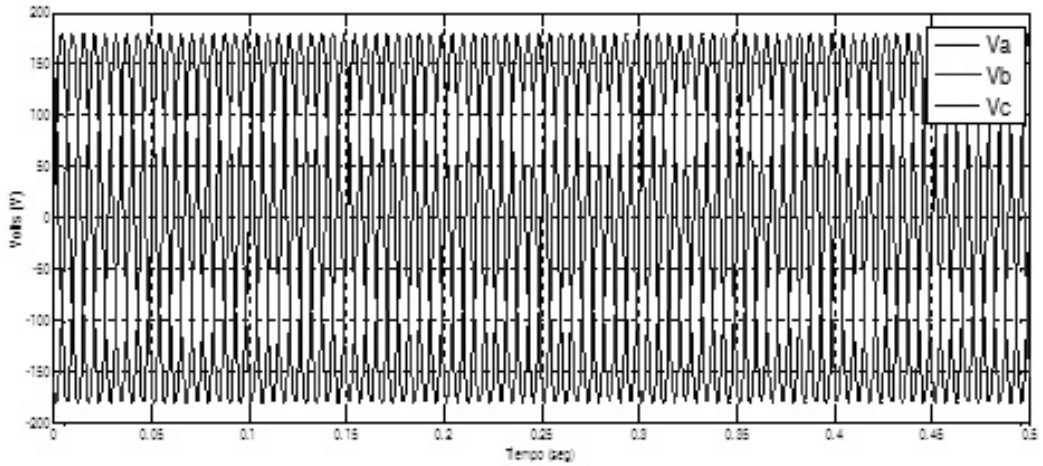

(a)

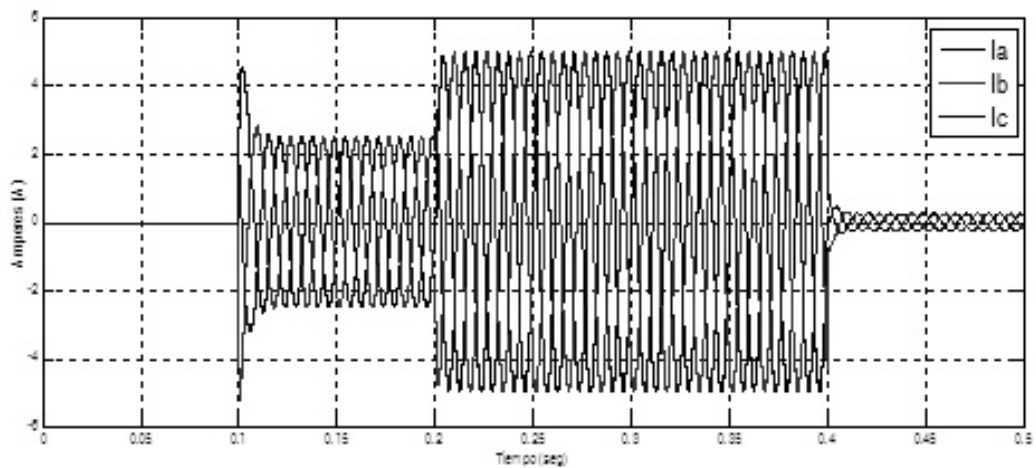

(b)

Fig. 12 Respuesta dinámica del voltaje y corriente ante variaciones de la carga. (a) Voltaje. (b) Corriente. 


\section{Referencias}

Ackermann, T. (2005). Wind Power in Power Systems. England, Jonh Wiley \& Sons, Ltd.

Bialasiewicz, J. T. (2008). Renewable energy systems with photovoltaic power generators: operation and modeling, IEEE Trans. on Industrial Electronics, 55(7), 2752-2758.

Boldea, I. (2006). Variable Speed Generators, USA, CRC Press.

Esram, T. \& Chapman, P. L. (2007). Comparison of photovoltaic array maximum power point tracking techniques, IEEE Trans. on Energy Conversion, 22(2), 439449.

Golnaraghi, F. \& Kuo, B. C. (2010). Automatic Control Systems, Ed. Wiley, New Jersey, USA.

Hau, E. (2006). Wind Turbines, Fundamentals, Technologies, Applications, Economics, Berlin, Germany, Springer.

Master, G. (2004). Renewable and Efficient Electric Power System, New Jersey, USA, Wiley-Interscience.

Munteanu, I., Bratcu, I., Cutululis, N. \& Ceanga, E. (2008). Optimal Control of Wind Energy Systems, London, Springer.

Nehrir, M. H. \& Wang, C. (2009). Modeling and Control of Fuel Cell, Distributed Generation Applications, New Jersey, USA, John Wiley \& Sons, Inc.

Pejovic, P. (2007). Three-Phase Diode Rectifiers with Low Harmonics, New York, USA.

Utkin, V., Guldner, J. \& Shi, J. (2009). Sliding Mode Control in Electro-Mechanical Systems, USA, CRC Press.

Wu, B., Lang, Y., Zargari, N., \& Kouro, S. (2011). Power Conversion and Control of Wind Energy Systems, New Jersey, USA, John Wiley \& Sons.

Yazdani, A. \& Iravani, R. (2010). Voltage-Sourced Converters in Power Systems, Modeling, Control and Applications, New Jersey, USA, John Wiley \& Sons, Inc. 